Geometry 8 Topology Monographs

Volume 1: The Epstein birthday schrift

Pages 1-21

\title{
The mean curvature integral is invariant under bending
}

\author{
Frederic J Almgren JR \\ IGOR RIVIN
}

\begin{abstract}
Suppose $\mathcal{M}_{t}$ is a smooth family of compact connected two dimensional submanifolds of Euclidean space $E^{3}$ without boundary varying isometrically in their induced Riemannian metrics. Then we show that the mean curvature integrals

$$
\int_{\mathcal{M}_{t}} H_{t} d \mathcal{H}^{2}
$$

are constant. It is unknown whether there are nontrivial such bendings $\mathcal{M}_{t}$. The estimates also hold for periodic manifolds for which there are nontrivial bendings. In addition, our methods work essentially without change to show the similar results for submanifolds of $H^{n}$ and $S^{n}$, to wit, if $\mathcal{M}_{t}=\partial X_{t}$

$$
d \int_{\mathcal{M}_{t}} H_{t} d \mathcal{H}^{2}=-k n-1 d V\left(X_{t}\right)
$$

where $k=-1$ for $H^{3}$ and $k=1$ for $S^{3}$. The Euclidean case can be viewed as a special case where $k=0$. The rigidity of the mean curvature integral can be used to show new rigidity results for isometric embeddings and provide new proofs of some well-known results. This, together with far-reaching extensions of the results of the present note is done in the preprint [6]. Our result should be compared with the well-known formula of Herglotz (see [5], also [8] and [2]).
\end{abstract}

AMS Classification 53A07, 49Q15

Keywords Isometric embedding, integral mean curvature, bending, varifolds

\section{Introduction}

The underlying idea of this note is the following. Suppose $\mathcal{N}_{t}$ is a smoothly varying family of polyhedral solids having edges $\left\{E_{t}(k)\right\}_{k}$, and associated (signed) dihedral angles $\left\{\theta_{t}(k)\right\}_{k}$. According to a theorem of Schlafli [7]

$$
\sum_{k}\left|E_{t}(k)\right| \frac{d}{d t} \theta_{t}(k)=0 .
$$


In case edge length is preserved in the family, ie

$$
\frac{d}{d t}\left|E_{t}(k)\right|=0
$$

for each time $t$ and each $k$, then also (product rule)

$$
\frac{d}{d t} \sum_{k}\left|E_{t}(k)\right| \theta_{t}(k)=0
$$

Should the $\partial \mathcal{N}_{t}$ 's be polyhedral approximations to submanifolds $\mathcal{M}_{t}$ varying isometrically, one might regard

$$
\sum_{k}\left|E_{t}(k)\right| \theta_{t}(k)
$$

as a reasonable approximation to the mean curvature integrals

$$
\int_{\mathcal{M}_{t}} H_{t} d \mathcal{H}^{2}
$$

and expect

$$
\frac{d}{d t}\left|E_{t}(k)\right|
$$

to be small. Hence it is plausible that the mean curvature integrals of the $\mathcal{M}_{t}$ 's might be constant. In this note we show that that is indeed the case.

Examples such as the isometry pictured on page 306 of volume 5 of [8] show that the mean curvature integral is not preserved under discrete isometries.

Two comments are in order. The first is that it is very likely that there are no isometric bendings of hypersurfaces. One reason for the existence of the current work is to produce a tool for resolving this conjecture (as Herglotz' mean curvature variation formula can be used to give a simple proof of Cohn-Vossen's theorem on rigidity of convex hypersurfaces). Secondly, the main theorem can be viewed as a sort of dual bellows theorem (when the hypersurface in question lies in $H^{n}$ or $S^{n}$ ): as the surface is isometrically deformed, the volume of the polar dual stays constant. This should be contrasted with the usual bellows theorem recently proved by Sabitov, Connelly and Walz [4].

\section{Terminology and basic facts}

Our object in this section is to set up terminology for a family of manifolds varying smoothly through isometries. We consider triangulations of increasing fineness varying with the manifolds. To make possible our mean curvature analysis we associate integral varifolds with both the manifolds and the polyhedral surfaces determined by the triangulations. The mean curvature integral of interest is identified with (minus two times) the varifold first variation associated with the unit normal initial velocity vector field. 


\subsection{Terminology and facts for a static manifold $\mathcal{M}$}

2.1.1 We suppose that $\mathcal{M} \subset \mathbb{R}^{3}$ is a compact connected smooth two dimensional submanifold of $\mathbb{R}^{3}$ without boundary oriented by a smooth Gauss mapping $\mathbf{n}: \mathcal{M} \rightarrow \mathbb{S}^{2}$ of unit normal vectors.

2.1.2 $H: \mathcal{M} \rightarrow \mathbb{R}$ denotes half the sum of principal curvatures in direction $\mathbf{n}$ at points in $\mathcal{M}$ so that $H \mathbf{n}$ is the mean curvature vector field of $\mathcal{M}$.

2.1.3 We denote by $U$ a suitable neighborhood of $\mathcal{M}$ in $\mathbb{R}^{3}$ in which a smooth nearest point retraction mapping $\rho: U \rightarrow \mathcal{M}$ is well defined. The smooth signed distance function $\sigma: U \rightarrow \mathbb{R}$ is defined by requiring $p=\rho(p)+\sigma(p) \mathbf{n}(\rho(p))$ for each $p$. We set

$$
g=\nabla \sigma: U \rightarrow \mathbb{R}^{3}
$$

(so that $g \mid \mathcal{M}=\mathbf{n}$ ); the vector field $g$ is the initial velocity vector field of the deformation

$$
G_{t}: U \rightarrow \mathbb{R}^{3}, \quad G_{t}(p)=p+\operatorname{tg}(p) \quad \text { for } p \in U
$$

2.1.4 We denote by

$$
V=\mathbf{v}(\mathcal{M})
$$

the integral varifold associated with $\mathcal{M}[1,3.5]$. The first variation distribution of $V[1,4.1,4.2]$ is representable by integration [1, 4.3] and can be written

$$
\delta V=\mathcal{H}^{2}\llcorner\mathcal{M} \wedge(-2 H) \mathbf{n}
$$

$[1,4.3 .5]$ so that

$$
\delta V(g)=\left.\frac{d}{d t} \mathcal{H}^{2}\left(G_{t}(\mathcal{M})\right)\right|_{t=0}=-2 \int_{\mathcal{M}} g \cdot H \mathbf{n} d \mathcal{H}^{2}=-2 \int_{\mathcal{M}} H d \mathcal{H}^{2}
$$

here $\mathcal{H}^{2}$ denotes two dimensional Hausdorff measure in $\mathbb{R}^{3}$.

2.1.5 By a vertex $p$ in $\mathcal{M}$ we mean any point $p$ in $\mathcal{M}$. By an edge $\langle p q\rangle$ in $\mathcal{M}$ we mean any (unordered) pair of distinct vertexes $p, q$ in $\mathcal{M}$ which are close enough together that there is a unique length minimizing geodesic arc $\llbracket p q \rrbracket$ in $\mathcal{M}$ joining them; in particular $\langle p q\rangle=\langle q p\rangle$. For each edge $\langle p q\rangle$ we write $\partial\langle p q\rangle=\{p, q\}$ and call $p$ a vertex of edge $\langle p q\rangle$, etc. We also denote by $\overline{p q}$ the straight line segment in $\mathbb{R}^{3}$ between $p$ and $q$, ie the convex hull of $p$ and $q$. By a facet $\langle p q r\rangle$ in $\mathcal{M}$ we mean any (unordered) triple of distinct vertexes $p, q, r$ which are not collinear in $\mathbb{R}^{3}$ such that $\langle p q\rangle,\langle q r\rangle,\langle r p\rangle$ are edges in $\mathcal{M}$; in particular, $\langle p q r\rangle=\langle q p r\rangle=\langle r p q\rangle$, etc. For each facet $\langle p q r\rangle$ we write 
$\partial\langle p q r\rangle=\{\langle p q\rangle,\langle q r\rangle,\langle r p\rangle\}$ and call $\langle p q\rangle$ an edge of facet $\langle p q r\rangle$ and also denote by $\overline{p q r}$ the convex hull of $p, q, r$ in $\mathbb{R}^{3}$.

2.1.6 Suppose $0<\tau<1$ and $0<\lambda<1$. By a $\tau, \lambda$ regular triangulation $\mathcal{T}$ of $\mathcal{M}$ of maximum edge length $L$ we mean

(i) a family $\mathcal{T}_{2}$ of facets in $\mathcal{M}$, together with

(ii) the family $\mathcal{T}_{1}$ of all edges of facets in $\mathcal{T}_{2}$ together with

(iii) the family $\mathcal{T}_{0}$ of all vertexes of edges in $\mathcal{T}_{1}$

such that

(iv) $\overline{p q r} \subset U$ for each facet $\langle p q r\rangle$ in $\mathcal{T}_{2}$

(v) $\mathcal{M}$ is partitioned by the family of subsets

$$
\begin{aligned}
\left\{\rho(\overline{p q r} \sim(\overline{p q} \cup \overline{q r} \cup \overline{r q})):\langle p q r\rangle \in \mathcal{T}_{2}\right\} & \cup\left\{\rho(\overline{p q}) \sim\{p, q\}:\langle p q\rangle \in \mathcal{T}_{1}\right\} \\
& \cup\left\{\{p\}: p \in \mathcal{T}_{0}\right\}
\end{aligned}
$$

(vi) for facets $\langle p q r\rangle \in \mathcal{T}_{2}$ we have the uniform nondegeneracy condition: if we set $u=q-p$ and $v=r-p$ then

$$
\left|v-\left(\frac{u}{|u|} \cdot v\right) \frac{u}{|u|}\right| \geq \tau|v|
$$

(vii) $L=\sup \left\{|p-q|:\langle p q\rangle \in \mathcal{T}_{1}\right\}$

(viii) for edges in $\mathcal{T}_{1}$ we have the uniform control on the ratio of lengths:

$$
\inf \left\{|p-q|:\langle p q\rangle \in \mathcal{T}_{1}\right\} \geq \lambda L \text {. }
$$

2.1.7 Fact [3] It is a standard fact about the geometry of smooth submanifolds that there are $0<\tau<1$ and $0<\lambda<1$ such that for arbitrarily small maximum edge lengths $L$ there are $\tau, \lambda$ regular triangulations of $\mathcal{M}$ of maximum edge length $L$. We fix such $\tau$ and $\lambda$. We hereafter consider only $\tau, \lambda$ regular triangulations $\mathcal{T}$ with very small maximum edge length $L$. Once $L$ is small the triangles $\overline{p q r}$ associated with $\langle p q r\rangle$ in $\mathcal{T}_{2}$ are very nearly parallel with the tangent plane to $\mathcal{M}$ at $p$.

2.1.8 Associated with each facet $\langle p q r\rangle$ in $\mathcal{T}_{2}$ is the unit normal vector $\mathbf{n}(p q r)$ to $\overline{p q r}$ having positive inner product with the normal $\mathbf{n}(p)$ to $\mathcal{M}$ at $p$. 
2.1.9 Associated with each edge $\langle p q\rangle$ in $\mathcal{T}_{1}$ are exactly two distinct facets $\langle p q r\rangle$ and $\langle p q s\rangle$ in $\mathcal{T}_{2}$. We denote by

$$
\mathbf{n}(p q)=\frac{\mathbf{n}(p q r)+\mathbf{n}(p q s)}{|\mathbf{n}(p q r)+\mathbf{n}(p q s)|}
$$

the average normal vector at $\overline{p q}$.

For each $\langle p q\rangle$ we further denote by $\theta(p q)$ the signed dihedral angle at $\overline{p q}$ between the oriented plane directions of $\overline{p q r}$ and $\overline{p q s}$ which is characterized by the condition

$$
2 \sin \left(\frac{\theta(p q)}{2}\right) \mathbf{n}(p q)=V+W
$$

where

- $V$ is the unit exterior normal vector to $\overline{p q r}$ along edge $\overline{p q}$, so that, in particular,

$$
V \cdot(p-q)=V \cdot \mathbf{n}(p q r)=0
$$

- $W$ is the unit exterior normal vector to $\overline{p q s}$ along edge $\overline{p q}$.

One checks that

$$
\cos \theta(p q)=\mathbf{n}(p q r) \cdot \mathbf{n}(p q s) .
$$

Finally for each $\langle p q\rangle$ we denote by

$$
g(p q)=|p-q|^{-1} \int_{\overline{p q}} g d \mathcal{H}^{1} \in \mathbb{R}^{3}
$$

the $\overline{p q}$ average of $g$; here $\mathcal{H}^{1}$ is one dimensional Hausdorff measure in $\mathbb{R}^{3}$.

2.1.10 Associated with our triangulation $\mathcal{T}$ of $\mathcal{M}$ is the polyhedral approximation

$$
\mathcal{N}[\mathcal{T}]=\cup\left\{\overline{p q r}:\langle p q r\rangle \in \mathcal{T}_{2}\right\}
$$

and the integral varifold

$$
V[\mathcal{T}]=\sum_{\langle p q r\rangle \in \mathcal{T}_{2}} \mathbf{v}(\overline{p q r})=\mathbf{v}(\mathcal{N}(\mathcal{T}))
$$

whose first variation distribution is representable by integration

$$
\delta V[\mathcal{T}]=\sum_{\langle p q\rangle \in \mathcal{T}_{1}} \mathcal{H}^{1}\left\llcorner\overline{p q} \wedge\left[2 \sin \left(\frac{\theta(p q)}{2}\right)\right] \mathbf{n}(p q)\right.
$$

$[1,4.3 .5]$ so that

$$
\delta V[\mathcal{T}](g)=\sum_{\langle p q\rangle \in \mathcal{T}_{1}}[|p-q|]\left[2 \sin \left(\frac{\theta(p q)}{2}\right)\right][\mathbf{n}(p q) \cdot g(p q)] .
$$




\subsection{Terminology and facts for a flow of manifolds $\mathcal{M}_{t}$}

2.2.1 As in 2.1.1 we suppose that $\mathcal{M} \subset \mathbb{R}^{3}$ is a compact connected smooth two dimensional submanifold of $\mathbb{R}^{3}$ without boundary oriented by a smooth Gauss mapping $\mathbf{n}: \mathcal{M} \rightarrow \mathbb{S}^{2}$ of unit normal vectors. We suppose additionally that $\varphi:(-1,1) \times \mathcal{M} \rightarrow \mathbb{R}^{3}$ is a smooth mapping with $\varphi(0, p)=p$ for each $p \in \mathcal{M}$. For each $t$ we set

$$
\varphi[t]=\varphi(t, \cdot): \mathcal{M} \rightarrow \mathbb{R}^{3} \quad \text { and } \quad \mathcal{M}_{t}=\varphi[t](\mathcal{M}) .
$$

Our principal assumption is that, for each $t$, the mapping $\varphi[t]: \mathcal{M} \rightarrow \mathcal{M}_{t}$ is an orientation preserving isometric imbedding (of Riemannian manifolds). In particular, each $\mathcal{M}_{t} \subset \mathbb{R}^{3}$ is a compact connected smooth two dimensional

submanifold of $\mathbb{R}^{3}$ without boundary oriented by a smooth Gauss mapping $\mathbf{n}_{\mathbf{t}}: \mathcal{M}_{t} \rightarrow \mathbb{S}^{2}$ of unit normal vectors.

2.2.2 As in 2.1.2, for each $t$, we denote by $H_{t} \mathbf{n}_{t}$ the mean curvature vector field of $\mathcal{M}_{t}$.

2.2.3 As in 2.1.3, for each $t$ we denote by $U_{t}$ a suitable neighborhood of $\mathcal{M}_{t}$ in $\mathbb{R}^{3}$ in which a smooth nearest point retraction mapping $\rho_{t}: U_{t} \rightarrow \mathcal{M}_{t}$ is well defined together with smooth signed distance function $\sigma_{t}: U_{t} \rightarrow \mathbb{R}$; also we set $g[t]=\nabla \sigma_{t}: U_{t} \rightarrow \mathbb{R}^{3}$ as an initial velocity vector field.

2.2.4 By a convenient abuse of notation we assume that we can define a smooth map

$$
\begin{gathered}
\varphi:(-1,1) \times U_{0} \rightarrow \mathbb{R}^{3} \\
\varphi(t, p)=\varphi\left(t, \rho_{0}(p)+\sigma_{0}(p) \mathbf{n}_{0}(\rho(p))=\varphi\left(t, \rho_{0}(p)\right)+\sigma_{0}(p) \mathbf{n}_{t}\left(\rho_{0}(p)\right)\right.
\end{gathered}
$$

for each $t$ and $p$. With $\varphi[t]=\varphi(t, \cdot)$ we have $\varphi[0]=\mathbf{1}_{U_{0}}$ and, additionally, $\sigma_{0}(p)=\sigma_{t}(\varphi[t](p))$. We further assume that

$$
U_{t}=\varphi[t] U_{0}
$$

for each $t$.

2.2.5 Fact If we replace our initial $\varphi[t]: \mathcal{M} \rightarrow \mathbb{R}^{3}$ 's by $\varphi[\mu t]$ for large enough $\mu$ (equivalently, restrict times $t$ to $-1 / \mu<t<1 / \mu$ ) and decrease the size of $U_{0}$ then the extended $\varphi[t]: U_{0} \rightarrow \mathbb{R}^{3}$ 's will exist. Such restrictions do not matter in the proof of our main assertion, since it is local in time and requires only small neighborhoods of the $\mathcal{M}_{t}$ 's.

2.1.6 As in 2.1.4, for each $t$ we denote by

$$
V_{t}=\mathbf{v}\left(\mathcal{M}_{t}\right)
$$


the integral varifold associated with $\mathcal{M}_{t}$.

2.2.7 We fix $0<\tau<1 / 2$ and $0<\lambda<1 / 2$ as in 2.1.7 and fix $2 \tau, 2 \lambda$ regular triangulations $\mathcal{T}(1), \mathcal{T}(2), \mathcal{T}(3), \ldots$ of $\mathcal{M}$ having maximum edge lengths $L(1), L(2), L(3) \ldots$ respectively with $\lim _{j \rightarrow \infty} L(j)=0$. For each $j$, the vertexes of $\mathcal{T}(j)$ are denoted $\mathcal{T}_{0}(j)$, the edges are denoted $\mathcal{T}_{1}(j)$, and the facets are denoted $\mathcal{T}_{2}(j)$. For all large $j$ and each $t$ we have triangulations $\mathcal{T}(1, t), \mathcal{T}(2, t), \mathcal{T}(3, t), \ldots$ of $\mathcal{M}_{t}$ as follows. With notation similar to that above we specify, for each $j$ and $t$,

$$
\begin{gathered}
\mathcal{T}_{0}(j, t)=\left\{\varphi[t](p): p \in \mathcal{T}_{0}(j)\right\}, \quad \mathcal{T}_{1}(j, t)=\left\{\langle\varphi[t](p) \varphi[t](q)\rangle:\langle p q\rangle \in \mathcal{T}_{1}(j)\right\}, \\
\mathcal{T}_{2}(j, t)=\left\{\langle\varphi[t](p) \varphi[t](q) \varphi[t](r)\rangle:\langle p q r\rangle \in \mathcal{T}_{2}(j)\right\} .
\end{gathered}
$$

2.2.8 Fact If we replace $\varphi[t]$ by $\varphi[\mu t]$ for large enough $\mu$ (equivalently, restrict times $t$ to $-1 / \mu<t<1 / \mu)$ then $\mathcal{T}(1, t), \mathcal{T}(2, t), \mathcal{T}(3, t), \ldots$ will a sequence of $\tau, \lambda$ regular triangulations of $\mathcal{M}$ with maximum edge lengths $L(j, t)$ converging to 0 uniformly in time $t$ as $j \rightarrow \infty$. Such restrictions do not matter in the proof of our main assertion, since it is local in time. We assume this has been done, if necessary, and that each of the triangulations $\mathcal{T}(j, t)$ is $\tau, \lambda$ regular with maximum edge lengths $L(j, t)$ converging to 0 as indicated.

2.2.9 As in 2.1.8 we associate with each $j, t$, and $\langle p q r\rangle \in \mathcal{T}_{2}(j)$ a unit normal vector $\mathbf{n}[t, j](p q r)$ to $\overline{\varphi[t](p) \varphi[t](q) \varphi[t](r)}$. As in 2.1.9 we associate with each $j, t$, and $\langle p q\rangle \in \mathcal{T}_{1}(j)$ an average normal vector $\mathbf{n}[t, j](p q)$ at $\overline{\varphi[t](p) \varphi[t](q)}$ and a signed dihedral angle $\theta[t, j](p q)$ at $\overline{\varphi[t](p) \varphi[t](q)}$ and the $\frac{\varphi[t](p) \varphi[t](q)}{\varphi[t]}$ average $g[t, j](p q)$ of $g[t]$.

2.2.10 As in 2.1.10 we associate with each triangulation $\mathcal{T}(j, t)$ of $\mathcal{M}_{t}$ a polyhedral approximation $\mathcal{N}[\mathcal{T}(j, t)]$ and an integral varifold

$$
V[\mathcal{T}(j, t)]=\mathbf{v}(\mathcal{N}[\mathcal{T}(j, t)])=\sum_{\langle p q r\rangle \in \mathcal{T}_{1}(j)} \mathbf{v}(\overline{\varphi[t](p) \varphi[t](q) \varphi[t](r)})
$$

with first variation distribution

$\delta V[\mathcal{T}(j, t)]=\sum_{\langle p q\rangle \in \mathcal{T}_{1}(j)} \mathcal{H}^{1}\left\llcorner[\overline{\varphi[t]) p) \varphi[t](q)}] \wedge\left[2 \sin \left(\frac{\theta[t, j](p q)}{2}\right)\right] \mathbf{n}[t, j](p q)\right.$. 
so that

$$
\begin{aligned}
& \delta V[\mathcal{T}(j, t)](g[t]) \\
& =\sum_{\langle p q\rangle \in \mathcal{T}_{1}(j)}[|\varphi[t](p)-\varphi[t](q)|]\left[2 \sin \left(\frac{\theta[t, j](p q)}{2}\right)\right][\mathbf{n}[t, j](p q) \cdot g[t, j](p q)] .
\end{aligned}
$$

2.2.11 The quantity we wish to show is constant in time is

$$
\int_{\mathcal{M}_{t}} H_{t} d \mathcal{H}^{2}=-\left(\frac{1}{2}\right) \delta V_{t}(g[t]) .
$$

Since, for each time $t$,

$$
V_{t}=\lim _{j \rightarrow \infty} V[\mathcal{T}(j, t)] \quad \text { (as varifolds) }
$$

we know, for each $t$,

$$
\delta V_{t}(g[t])=\lim _{j \rightarrow \infty} \delta V[\mathcal{T}(j, t)](g[t]) .
$$

We are thus led to seek to estimate

$$
\frac{d}{d t} \delta V[\mathcal{T}(j, t)](g[t])
$$

using the formula in 2.2.10. A key equality it provided by Schlafli's theorem mentioned above which, in the present terminology, asserts for each $j$ and $t$,

$$
\sum_{\langle p q\rangle \in \mathcal{T}_{1}(j)}[|\varphi[t](p)-\varphi[t](q)|] \frac{d}{d t}[\theta[t, j](p q)]=0 .
$$

2.2.12 Fact Since, for each $\langle p p q\rangle$ in $\mathcal{T}_{2}(j), \partial\langle p q r\rangle$ consists of exactly three edges, and, for each $\langle p q\rangle$ in $\mathcal{T}_{1}(j)$, there are exactly two distinct facets $\langle p q r\rangle$ in $\mathcal{T}_{2}(j)$ for which $\langle p q\rangle \in \partial\langle p q r\rangle$ we infer that, for each $j$,

$$
\operatorname{card}\left[\mathcal{T}_{1}(j)\right]=\frac{3}{2} \operatorname{card}\left[\mathcal{T}_{2}(j)\right]
$$

We then use the $\tau, \lambda$ regularity of the the $\mathcal{T}(j)$ 's to check that that, for each time $t$ and each $\langle p p q\rangle$ in $\mathcal{T}_{2}(j)$ the following four numbers have bounded ratios (independent of $j, t$, and $\langle p p q\rangle$ ) with each other

$$
\mathcal{H}^{2}(\overline{\varphi[t](p) \varphi[t](q) \varphi[t](r)}), \quad|\varphi[t](p)-\varphi[t](q)|^{2}, \quad L(j, t)^{2}, \quad L(j)^{2} .
$$

Since

$$
\lim _{j \rightarrow \infty} \mathcal{H}^{2}(\mathcal{N}[j, t])=\mathcal{H}^{2}\left(\mathcal{M}_{t}\right)=\mathcal{H}^{2}(\mathcal{M})
$$

we infer

$$
\sup _{j} \sum_{\langle p q\rangle \in \mathcal{T}_{1}(j)} L(j)^{2}<\infty, \quad \lim _{j \rightarrow \infty} \sum_{\langle p q\rangle \in \mathcal{T}_{1}(j)} L(j)^{3}=0 .
$$




\section{Modifications of the flow}

\subsection{Justification for computing with modified flows}

As indicated in 2.2, we wish to estimate the time derivatives of

$$
\begin{aligned}
& \delta V[\mathcal{T}(j, t)](g[t]) \\
& =\sum_{\langle p q\rangle \in \mathcal{T}_{1}(j)}[|\varphi[t](p)-\varphi[t](q)|]\left[2 \sin \left(\frac{\theta[t, j](p q)}{2}\right)\right][\mathbf{n}[t, j](p q) \cdot g[t, j](p q)] .
\end{aligned}
$$

In each of the $\langle p q\rangle$ summands, each of the three factors

$$
[|\varphi[t](p)-\varphi[t](q)|], \quad\left[2 \sin \left(\frac{\theta[t, j](p q)}{2}\right)\right], \quad[\mathbf{n}[t, j](p q) \cdot g[t, j](p q)]
$$

is an intrinsic geometric quantity (at each time) whose value does not change under isometries of the ambient $\mathbb{R}^{3}$. With $\langle p q r\rangle$ and $\langle p q s\rangle$ denoting the two facets sharing edge $\langle p q\rangle$, we infer that each of the factors depends at most on the relative positions of $\varphi[t](p), \varphi[t](q), \varphi[t](r), \varphi[t](s)$ and $\varphi[t] \mathcal{M}$. Suppose $\psi:(-1,1) \times \mathbb{R}^{3} \rightarrow \mathbb{R}^{3}$ is continuously differentiable, and for each $t$, the function $\psi[t]=\psi(t, \cdot): \mathbb{R}^{3} \rightarrow \mathbb{R}^{3}$ is an isometry. Suppose further, we set

$$
\varphi^{*}(t, p)=\psi(t, \varphi(t, p)), \quad \varphi^{*}[t]=\varphi^{*}(t, \cdot)
$$

for each $t$ and $p$ so that $\varphi^{*}[t]=\psi[t] \circ \varphi[t]$. If we replace $\mathcal{M}$ by $\mathcal{M}^{*}=\psi[0] \mathcal{M}$ and $\varphi$ by $\varphi^{*}$ then we could follow the procedures of 2.1 and 2.2 to construct triangulations and polyhedral approximations $\mathcal{T}^{*}[j, t]$ and varifolds $V^{*}$, etc. with

$$
\delta V[\mathcal{T}(j, t)](g[t])=\delta V^{*}\left[\mathcal{T}^{*}(j, t)\right]\left(g^{*}[t]\right) .
$$

Not only do we have equality in the sum, but, for each $\langle p q\rangle$ the corresponding summands are identical numerically. Hence, in evaluating $\delta V[\mathcal{T}(j, t)](g[t])$ we are free to (and will) use a different $\psi$ and $\varphi^{*}$ for each summand.

\subsection{Conventions for derivatives}

Suppose $W$ is an open subset of $\mathbb{R}^{M}$ and $f=\left(f^{1}, f^{2}, \ldots, f^{N}\right): W \rightarrow \mathbb{R}^{N}$ is $K$ times continuously differentiable. We denote by

$$
\left\|\left|D^{K} f \|\right|\right.
$$

the supremum of the partial derivatives

$$
\frac{\partial^{k} f^{K}}{\partial x_{i(1)} \partial x_{i(2)} \ldots \partial x_{i(K)}}(p)
$$

corresponding to all points $p \in W$, all $\{i(1), i(2), \ldots, i(K)\} \subset\{1, \ldots, M\}$ and $k=1, \ldots, N$, all choices of orthonormal coordinates $\left(x_{1}, \ldots, x_{M}\right)$ for $\mathbb{R}^{M}$ and all choices of orthonormal coordinates $\left(y_{1}, \ldots, y_{N}\right)$ for $\mathbb{R}^{N}$. 


\subsection{Conventions for inequalities}

In making various estimates we will use use the largest edge length of the $j$ th triangulation, typically called $L$, and a general purpose constant $C$. The constant $C$ will have different values in different contexts (even in the same formula). What is implied is that, with $\mathcal{M}$ and $\varphi$ fixed, the constants $C$ can be chosen independent of the level of triangulation (once it is fine enough) and independent of time $t$ and independent of the various modifications of our flow which are used in obtaining our estimates. As a representative example of our terminology, the expression

$$
A=B \pm C L^{2}
$$

means

$$
-C L^{2} \leq A-B \leq C L^{2} .
$$

\subsection{Fixing a vertex at the origin}

Suppose $p$ is a vertex in $\mathcal{M}$ and

$$
\varphi_{*}(-1,1) \times U_{0} \rightarrow \mathbb{R}^{3}, \quad \varphi_{*}(t, q)=\varphi(t, q)-\varphi(t, p) \quad \text { for each } q .
$$

Then $\varphi^{*}(t, p)=(0,0,0)$ for each $t$. One checks, for $K=0,1,2,3$ that

$$
\left\|\left|D^{K} \varphi^{*}\||\leq 2|\| D^{K} \varphi\|\|, \quad\left\|\left|D^{K} \varphi^{*}[t]\|\|=\left\|D^{K} \varphi[t]\right\|\right|\right.\right.\right.
$$

for each $t$.

\subsection{Mapping a frame to the basis vectors}

Suppose $(0,0,0) \in \mathcal{M}$ and that $\mathbf{e}_{1}$ and $\mathbf{e}_{2}$ are tangent to $\mathcal{M}$ at $(0,0,0)$. Suppose also $\varphi(t, 0,0,0)=(0,0,0)$ for each $t$. Then the mapping $\varphi^{*}$ given by setting

$$
\varphi^{*}[t]=\left(\begin{array}{lll}
\frac{\partial \varphi^{1}}{\partial x_{1}}(t, 0,0,0) & \frac{\partial \varphi^{2}}{\partial x_{1}}(t, 0,0,0) & \frac{\partial \varphi^{3}}{\partial x_{1}}(t, 0,0,0) \\
\frac{\partial \varphi^{1}}{\partial x_{2}}(t, 0,0,0) & \frac{\partial \varphi^{2}}{\partial x_{2}}(t, 0,0,0) & \frac{\partial \varphi^{3}}{\partial x_{2}}(t, 0,0,0) \\
\frac{\partial \varphi^{1}}{\partial x_{3}}(t, 0,0,0) & \frac{\partial \varphi^{2}}{\partial x_{3}}(t, 0,0,0) & \frac{\partial \varphi^{3}}{\partial x_{3}}(t, 0,0,0)
\end{array}\right) \circ \varphi[t]
$$

satisfies

$$
\varphi^{*}[t](0,0,0)=(0,0,0), \quad D \varphi^{*}[t](0,0,0)=\mathbf{1}_{\mathbf{R}^{3}}
$$

with

$$
||\left|D^{K} \varphi^{*}[t]\right|||=||\left|D^{K} \varphi[t]\right|||
$$

for each $K=1,2,3$ and each $t$, and

$$
\left\|\frac{\partial \varphi^{*}}{\partial t}(t, \cdot)\right\||| \leq 3\left(\left\|D^{0} \varphi\left|\|\cdot\| D^{2} \varphi\right|\right\|+\left\|\left|D^{1} \varphi[t] \|\right|^{2}\right) .\right.
$$


3.6 Theorem There is $C<\infty$ such that the following is true for all sufficiently small $\delta>0$. Suppose $\gamma_{0}:[0, \delta] \rightarrow \mathcal{M}$ is an arc length parametrization of a length minimizing geodesic in $\mathcal{M}$ and set

$$
\gamma(s, t)=\varphi[t]\left(\gamma_{0}(s)\right) \quad \text { for each } s \text { and } t
$$

so that $s \rightarrow \gamma(s, t)$ is an arc length parametrization of a geodesic in $\mathcal{M}_{t}$. We also set

$$
r(s, t)=|\gamma(0, t)-\gamma(s, t)| \quad \text { for each } s \text { and } t
$$

and, for (fixed) $0<R<\delta$, consider

$$
r(R, t)=|\gamma(0, t)-\gamma(R, t)| \quad \text { for each } t .
$$

Then

$$
\frac{d}{d t} r(R, t)= \pm C R^{2}
$$

and

$$
\lim _{R \downarrow 0} R^{-1} \frac{d}{d t} r(R, t)=0 .
$$

Proof We will show

$$
\left.\frac{d}{d t} r(R, t)\right|_{t=0}= \pm C R^{2}
$$

Step 1 Replacing $\varphi(t, p)$ by $\varphi^{*}(t, p)=\varphi(t, p)-\varphi\left(t, \gamma_{0}(0)\right)$ as in 3.4 if necessary we assume without loss of generality that $\gamma(0, t)=(0,0,0)$ for each $t$.

Step 2 Rotating coordinates if necessary we assume without loss of generality that $\mathbf{e}_{1}$ and $\mathbf{e}_{2}$ are tangent to $\mathcal{M}_{0}$ at $(0,0,0)$ and that $\gamma_{0}^{\prime}(0)=\mathbf{e}_{1}$

Step 3 Rotating coordinates as time changes as in 3.5 if necessary we assume without loss of generality that $D \varphi[t](0,0,0)=\mathbf{1}_{\mathbf{R}^{3}}$ for each $t$.

Step 4 We define

$$
X(s, t)=\gamma(s, t) \cdot \mathbf{e}_{1}, \quad Y(s, t)=\gamma(s, t) \cdot \mathbf{e}_{2}, \quad Z(s, t)=\gamma(s, t) \cdot \mathbf{e}_{3}
$$

so that

$$
\gamma(s, t)=(X(s, t), Y(s, t), Z(s, t))
$$

and estimate for each $s$ and $t$ :

(a) $X(0, t)=Y(0, t)=Z(0, t)=0$ (by step 1$)$ 
(b) $X_{t}(0,0)=Y_{t}(0,0)=Z_{t}(0,0)=0$

(c) $X_{s}(s, t)^{2}+Y_{s}(s, t)^{2}+Z_{s}(s, t)^{2}=1$

(d) $X_{s}(s, t)= \pm 1, Y_{s}(s, t)= \pm 1, Z_{s}(s, t)= \pm 1$

(e) $1 / 2 \leq r(s, t) /|s| \leq 1$ (since $\delta$ is small)

(f) $X(s, 0)= \pm C s, Y(s, 0)= \pm C s, Z(s, 0)= \pm C s$

(g) $X_{s}(0, t)=X_{s}(0,0), Y_{s}(0, t)=Y_{s}(0,0), Z_{s}(0, t)=Z_{s}(0,0)$ (by step 3$)$

(h) $X_{s t}(0,0)=Y_{s t}(0,0)=Z_{s t}(0,0)=0$

(i) $\quad X_{s t}(s, 0)=X_{s t}(0,0)+\int_{0}^{s} X_{s s t}(\eta, 0) d \eta=0 \pm s \sup \left|X_{s s t}\right|= \pm C s$,

$$
Y_{s t}(s, 0)= \pm C s, \quad Z_{s t}(s, 0)= \pm C s
$$

(j)

$$
\begin{gathered}
X_{t}(s, 0)=X_{t}(0,0)+\int_{0}^{s} X_{s t}(\eta, 0) d \eta=0 \pm C s^{2}, \\
Y_{t}(s, 0)= \pm C s^{2}, \quad Z_{t}(s, 0)= \pm C s^{2}
\end{gathered}
$$

(k) $r^{2}=X^{2}+Y^{2}+Z^{2}$

$$
r r_{s}=X X_{s}+Y Y_{s}+Z Z_{s}, \quad r_{s}=\frac{1}{r}\left(X X_{s}+Y Y_{s}+Z Z_{s}\right)
$$

$(\mathrm{m})$

$$
r r_{t}=X X_{t}+Y Y_{t}+Z Z_{t}, \quad r_{t}=\frac{1}{r}\left(X X_{t}+Y Y_{t}+Z Z_{t}\right)
$$

(n) $r_{s} r_{t}+r r_{s t}=X_{s} X_{t}+X X_{s t}+Y_{s} Y_{t}+Y Y_{s t}+Z_{s} Z_{t}+Z Z_{s t}$

(o) evaluating (n) at $t=0, r>0$ we see

$$
\begin{aligned}
\frac{1}{r(s, 0)^{2}}(( \pm C s)( \pm 1))\left(( \pm C s)\left( \pm C s^{2}\right)\right) & +r(s, 0) r_{s t}(s, 0) \\
= & ( \pm 1)\left( \pm C s^{2}\right)+( \pm C s)( \pm C s)
\end{aligned}
$$

(p) $r_{s t}(s, 0)= \pm C s$

(q) $\quad r_{t}(R, 0)=r_{t}(0,0)+\int_{0}^{R} r_{s t}(s, 0) d s=0+\int_{0}^{R} \pm C s d s= \pm C R^{2}$. 
3.7 Corollary Suppose triangulation $\mathcal{T}(j)$ has maximum edge length $L=$ $L(j)$ and $\langle p q\rangle$ is an edge in $\mathcal{T}_{1}(j)$. Then, for each $t$,

$$
|\varphi[t](p)-\varphi[t](q)|= \pm C L \quad \text { and } \quad \frac{d}{d t}|\varphi[t](p)-\varphi[t](q)|= \pm C L^{2}
$$

\subsection{Stabilizing the facets of an edge}

Suppose $\mathcal{T}(j)$ is a triangulation with maximum edge length $L=L(j)$ and that $\langle A B C\rangle,\langle A C D\rangle$ are facets in $\mathcal{T}_{2}(j)$ as illustrated

$$
(0,0,0)=A \begin{gathered}
\swarrow^{D=(e, f, 0)} \\
\searrow \\
B=(a, b, c)
\end{gathered} \nearrow^{\longleftrightarrow} C=(d, 0,0) .
$$

Interchanging $B$ and $D$ if necessary we assume without loss of generality the the average normal $\mathbf{n}[0, A C]$ to $\mathcal{M}_{0}$ at $A$ has positive inner product with $(C-A) \times(D-A)$.

1) Fixing $A$ at the origin Modifying $\varphi$ if necessary as in 3.4 if necessary we can assume without loss of generality that $\varphi[t](A)=(0,0,0)$ for each $t$. As indicated there, various derivative bounds are increased by, at most, a controlled amount.

2) Convenient rotations We set $u(t)=\varphi[t](C), \quad v(t)=\varphi[t](D)$ and use the Gramm-Schmidt orthonormalization process to construct

$$
U(t)=\frac{u(t)}{|u(t)|}, \quad V(t)=\frac{v(t)-v(t) \cdot U(t) U(t)}{|v(t)-v(t) \cdot U(t) U(t)|}, \quad W(t)=U(t) \times V(t) .
$$

One uses the mean value theorem in checking

$$
\left\|\mid D^{K} U(t)\right\| \leq C\left(\sum_{j=0}^{K+1}\left\|\mid D^{j} \varphi\right\|\right), \quad \text { etc }
$$

for each $K=0,1,2$. We denote by $Q(t)$ the orthogonal matrices having columns equal to $U(t), V(t), W(t)$ respectively (which is the inverse matrix to its transpose). Replacing $\varphi_{t}$ by $Q(t) \circ \varphi_{t}$ if necessary, we assume without loss of generality that there are functions $a(t), b(t), c(t), d(t), e(t), f(t)$, such that

$$
\begin{aligned}
\varphi[t](A) & =(0,0,0), \quad \varphi[t](B)=(a(t), b(t), c(t)), \\
\varphi[t](C) & =(d(t), 0,0), \quad \varphi[t](D)=(e(t), f(t), 0) .
\end{aligned}
$$


We assume without loss of generality the existence of functions $F[t](x, y)$ defined for $(x, y)$ near $(0,0)$ such that, near $(0,0,0)$ our manifold $\mathcal{M}_{t}$ is the graph of $F[t]$. In particular,

$$
c(t)=F[t](a(t), b(t)) .
$$

We assert that if $|p| \leq C L$, then

$$
|F[t](p)| \leq C L^{2}, \quad|\nabla F[t](p)| \leq C L
$$

To see this, first we note that $F[t](A)=F[t](C)=F[t](D)=0$. Next we invoke Rolle's theorem to conclude the existence of $c_{1}$ on segment $A D$ and $c_{2}$ on segment $C D$ such

$$
\left\langle\frac{D-A}{|D-A|}, D F[t]\left(c_{1}\right)\right\rangle=0=\left\langle\frac{D-C}{|D-C|}, D F[t]\left(c_{2}\right)\right\rangle .
$$

Since $|p| \leq C L$ we infer

$$
\left\langle\frac{D-A}{|D-A|}, D F[t](p)\right\rangle= \pm C L, \quad\left\langle\frac{D-C}{|D-C|}, D F[t](p)\right\rangle= \pm C L .
$$

In view of 2.1.6(vi)(vii)(viii) and 2.2.7 we infer that $\mathbf{e}_{1}$ and $\mathbf{e}_{2}$ are bounded linear combinations of $(D-A) /|D-A|$ and $(D-C) /|D-C|$ from which we conclude that $|\nabla F[t](p)| \leq C L$. This in turn implies that $|F[t](p)| \leq C L^{2}$ as asserted.

Since

we infer

$$
\frac{\partial}{\partial t} F[t](0,0)=0
$$

$$
\frac{\partial}{\partial t} F[t](p)= \pm C L
$$

and since

we infer

$$
\frac{\partial}{\partial t}\left(\varphi[t](A) \cdot \mathbf{e}_{3}\right)=0
$$

$$
c^{\prime}(t)=\frac{\partial}{\partial t} F[t](a(t), b(t))=\frac{\partial}{\partial t}\left(\varphi[t](B) \cdot \mathbf{e}_{3}\right)= \pm C L .
$$

3.9 Proposition Let $L, A, B, C, D, a, b, c, d, e, f$ be as in 3.8. Then

(1) $a^{\prime}(t)= \pm C L^{2}$

(2) $\quad b^{\prime}(t)= \pm C L^{2}$

(3) $c^{\prime}(t)= \pm C L$

(4) $d^{\prime}(t)= \pm C L^{2}$

(5) $e^{\prime}(t)= \pm C L^{2}$

(6) $f^{\prime}(t)= \pm C L^{2}$. 
Proof According to 3.7, if $r(t)$ denotes the distance between the endpoints of an edge of arc length $L$ at time $t$, then

$$
r^{\prime}(t)= \pm C L^{2}
$$

(i) We invoke 3.7 directly to infer (4) above.

(ii) We apply 3.7 to the distance between $(0,0,0)$ and $(e, f, 0)$ to infer

$$
\frac{d}{d t}\left(e^{2}+f^{2}\right)^{\frac{1}{2}}=\frac{\left(e e^{\prime}+f f^{\prime}\right)}{\left(e^{2}+f^{2}\right)^{\frac{1}{2}}}= \pm C L^{2}, \quad e e^{\prime}+f f^{\prime}= \pm C L^{3} .
$$

(iii) We apply 3.7 to the distance between $(d, 0,0)$ and $(e, f, 0)$ to infer

$$
\begin{gathered}
\frac{d}{d t}\left((e-d)^{2}+f^{2}\right)^{\frac{1}{2}}=\frac{\left.(e-d)\left(e^{\prime}-d^{\prime}\right)+f f^{\prime}\right)}{\left((e-d)^{2}+f^{2}\right)^{\frac{1}{2}}}= \pm C L^{2}, \\
(e-d)\left(e^{\prime}-d^{\prime}\right)+f f^{\prime}= \pm C L^{3} .
\end{gathered}
$$

We subtract the first inequality from the second to infer

$$
e d^{\prime}-d e^{\prime}+d d^{\prime}= \pm C L^{3}, \quad d e^{\prime} \pm C L^{3}, \quad e^{\prime}= \pm C L^{2} .
$$

Assertions (5) and (6) follow readily.

(iv) We apply 3.7 to the distance between $(0,0,0)$ and $(a, b, c)$ to infer

$$
\frac{d}{d t}\left(a^{2}+b^{2}+c^{2}\right)^{\frac{1}{2}}=\frac{\left(a a^{\prime}+b b^{\prime}+c c^{\prime}\right)}{\left(a^{2}+b^{2}+c^{2}\right)^{\frac{1}{2}}}= \pm C L^{2}, \quad a a^{\prime}+b b^{\prime}+c c^{\prime}= \pm C L^{3} .
$$

(v) We apply 3.7 to the distance between $(d, 0,0)$ and $(a, b, c)$ to infer

$$
\begin{gathered}
\frac{d}{d t}\left((a-d)^{2}+b^{2}+c^{2}\right)^{\frac{1}{2}}=\frac{\left((a-d)\left(a^{\prime}-d^{\prime}\right)+b b^{\prime}+c c^{\prime}\right)}{\left((a-d)^{2}+b^{2}+c^{2}\right)^{\frac{1}{2}}}= \pm C L^{2}, \\
(a-d)\left(a^{\prime}-d^{\prime}\right)+b b^{\prime}+c c^{\prime}= \pm C L^{3} .
\end{gathered}
$$

We subtract the first inequality form the second to infer

$$
a d^{\prime}-d a^{\prime}+d d^{\prime}= \pm C L^{3}, \quad d a^{\prime} \pm C L^{3}, \quad a^{\prime}= \pm C L^{2},
$$

which gives assertion (1).

(vi) We estimate from 3.8 that

$$
c=F[t](a, b)= \pm C L^{2}, \quad c^{\prime}=\frac{d}{d t} F[t](a, b)+\nabla F[t](a, b) \cdot\left(a^{\prime}, b^{\prime}\right)= \pm C L,
$$

which gives (3) above. We have also $c c^{\prime}= \pm C L^{3}$. We recall (iv) above and estimate

$$
a a^{\prime}+b b^{\prime}+c c^{\prime}= \pm C L^{3}, \quad b b^{\prime}= \pm C L^{3}, \quad b^{\prime}= \pm C L^{2},
$$

which is (2) above. 
3.10 Proposition Suppose $\mathcal{T}(j)$ is a triangulation with maximum edge length $L=L(j)$ and $\langle p q\rangle$ is an edge in $\mathcal{T}_{1}(j)$. Abbreviate $\theta(t)=\theta[t, j](p q)$. Then, for each $t$,

$$
\begin{gathered}
\theta(t)= \pm C L \\
2 \sin \left(\frac{\theta(t)}{2}\right)= \pm C L \\
\theta^{\prime}(t)= \pm C \\
\frac{d}{d t}\left[2 \sin \left(\frac{\theta(t)}{2}\right)\right]= \pm C \\
\frac{d}{d t}\left[2 \sin \left(\frac{\theta(t)}{2}\right)-\theta\right]= \pm C L^{2} .
\end{gathered}
$$

Proof Making the modifications of 3.8 if necessary, we assume without loss of generality (in the terminology there) that $\varphi[t](p)=A=(0,0,0), \varphi[t](q)=$ $C=(d(t), 0,0)$, and that there are $\left\langle p q B_{*}\right\rangle,\left\langle p q D_{*}\right\rangle \in \mathcal{T}_{2}(j)_{0}$ with $\varphi[t]\left(B_{*}\right)=$ $B=(a(t), b(t), c(t)), \varphi[t]\left(D_{*}\right)=D=(e(t), f(t), 0)$.

The unit normal to $\overline{A C D}$ is $(0,0,1)$ while the unit normal to $\overline{A B C}$ is

$$
\frac{(0,-c, b)}{\left(b^{2}+c^{2}\right)^{\frac{1}{2}}}
$$

so that $\quad \cos \theta=\frac{b}{\left(b^{2}+c^{2}\right)^{\frac{1}{2}}}$

$$
\sin \theta= \pm\left(1-\cos ^{2} \theta\right)^{\frac{1}{2}}= \pm\left(1-\frac{b^{2}}{b^{2}+c^{2}}\right)^{\frac{1}{2}}= \pm \frac{c}{\left(b^{2}+c^{2}\right)^{\frac{1}{2}}}= \pm C L
$$

in view of 3.8. Assertions (1) and (2) follow. We compute further

$$
(\sin \theta)^{\prime}=\cos \theta \theta^{\prime}= \pm \frac{\left(b^{2}+c^{2}\right)^{\frac{1}{2}} c^{\prime}-c \frac{b b^{\prime}+c c^{\prime}}{\left(b^{2}+c^{2}\right)^{\frac{1}{2}}}}{b^{2}+c^{2}}= \pm C
$$

in view of 3.9(1)(2)(3) and 3.8. Assertion (3) and (4) follow. Assertion (5) follows from differentiation and assertions (1) and (3). 
3.11 Proposition Suppose $\mathcal{T}(j)$ is a triangulation with maximum edge length $L=L(j)$ and $\langle p q\rangle$ is an edge in $\mathcal{T}_{1}(j)$. Then

(1) $\mathbf{n}[t, j](p q)=\left(0, \pm C L, 1 \pm C L^{4}\right)$

(2) $(d / d t)(\mathbf{n}[t, j](p q))=(0, \pm C, \pm C L)+( \pm C L, \pm C L, \pm C L)$

(3) $g[t, j](p q)=\left( \pm C L, \pm C L, 1 \pm C L^{2}\right)$

(4) $(d / d t) g[t, j](p q)=( \pm C, \pm C, 0)+( \pm C L, \pm C L, \pm C L)$

(5) $\mathbf{n}[t, j](p q) \cdot g[t, j](p q)=1 \pm C L^{2}$

(6) $\quad(d / d t)(\mathbf{n}[t, j](p q) \cdot g[t, j](p q))= \pm C L$

(7) $1-\mathbf{n}[t, j](p q) \cdot g[t, j](p q)= \pm C L^{2}$.

Proof We let $A, B, C, D, F[t], b(t), c(t), d(t)$ be as in 3.8. We abbreviate $\mathbf{n}=\mathbf{n}[t, j](p q)$ and estimate

$$
\begin{aligned}
\mathbf{n} & =\frac{(0,0,1)+(0,-c, b) /\left(b^{2}+c^{2}\right)^{\frac{1}{2}}}{\left|(0,0,1)+(0,-c, b) /\left(b^{2}+c^{2}\right)^{\frac{1}{2}}\right|} \\
& =\frac{\left(0,-c, b+\left(b^{2}+c^{2}\right)^{\frac{1}{2}}\right)}{2^{\frac{1}{2}}\left(b^{2}+c^{2}+b\left(b^{2}+c^{2}\right)^{\frac{1}{2}}\right)^{\frac{1}{2}}} .
\end{aligned}
$$

The first assertion follows from 3.8.1. We differentiate to conclude $\mathbf{n}^{\prime}=$

$$
\begin{gathered}
\frac{ \pm C L\left(0,-c^{\prime}, b^{\prime} \pm C\left(b b^{\prime}+c c^{\prime}\right) / L-(L / L)\left(b b^{\prime}+c c^{\prime} \pm b^{\prime} L+ \pm C(b / L)\left(b b^{\prime}+c c^{\prime}\right)\right.\right.}{ \pm L^{2}} \\
=(0, \pm C, \pm C L)+( \pm C L, \pm C L, \pm C L)
\end{gathered}
$$

in view of $3.9(2)(3)$. This is assertion (2).

We abbreviate $g=g[t, j](p q)$ and estimate

$$
\begin{aligned}
g & =\frac{1}{d(t)} \int_{0}^{d(t)} \frac{\left(-F[t]_{x},-F[t]_{y}, 1\right)}{\left|\left(-F[t]_{x},-F[t]_{y}, 1\right)\right|} \\
& =\frac{1}{d(t)} \int_{0}^{d(t)} \frac{\left(-F[t]_{x},-F[t]_{y}, 1\right)}{\left(\left(F[t]_{x}^{2} F[t]_{y}^{2}+1\right)^{\frac{1}{2}}\right.} .
\end{aligned}
$$


The third assertion follows from 3.8.1. We differentiate to estimate that $d g / d t$ equals

$$
\begin{aligned}
& \frac{-d^{\prime}}{d^{2}} \int_{0}^{d(t)} \frac{\left(-F[t]_{x},-F[t]_{y}, 1\right)}{\left(1+F[t]_{x}^{2}+F[t]_{y}^{2}\right)^{\frac{1}{2}}}+\frac{d^{\prime}}{d} \frac{\left(-F[t]_{x},-F[t]_{y}, 1\right)}{\left(1+F[t]_{x}^{2}+F[t]_{y}^{2}\right)^{\frac{1}{2}}} \\
& \quad+\frac{1}{d} \int_{0}^{d} \frac{ \pm C L\left(-F[t]_{t x},-F[t]_{t y}, 0\right)}{1+F[t]_{x}^{2}+F[t]_{y}^{2}} \\
& \quad-\frac{1}{d} \int_{0}^{d} \frac{\left(-F[t]_{x},-F[t]_{y}, 1\right)( \pm C / L)\left(F[t]_{x} F[t]_{t x}+F[t]_{y} F[t]_{t y}\right)}{1+F[t]_{x}^{2}+F[t]_{y}^{2}}= \\
& L( \pm C, \pm C, \pm C)+L( \pm C, \pm C, \pm C)+( \pm C, \pm C, 0)+L( \pm C, \pm C, \pm C)
\end{aligned}
$$

which gives assertion (4). Assertion (5) follows from assertions (1) and (3). Assertion (6) follows from assertions (1), (2), (3), (4) and integration by parts. Assertion (7) follows from assertions (1) and (3).

\section{Constancy of the mean curvature integral}

\subsection{The derivative estimates}

Suppose triangulation $\mathcal{T}(j)$ has maximum edge length $L=L(j)$. We recall from 2.2.10 that

$$
\begin{aligned}
& \delta V[\mathcal{T}(j, t)](g[t]) \\
& =\sum_{\langle p q\rangle \in \mathcal{T}_{1}(j)}[|\varphi[t](p)-\varphi[t](q)|]\left[2 \sin \left(\frac{\theta[t, j](p q)}{2}\right)\right][\mathbf{n}[t, j](p q) \cdot g[t, j](p q)]
\end{aligned}
$$

and we estimate, for each $t$ that

$$
\begin{aligned}
& \frac{d}{d t}\left(\delta V\left[\mathcal{T}(j)_{t}\right](g[t])\right) \\
& =\sum_{\langle p q\rangle \in \mathcal{T}_{1}(j)}[|\varphi[t](p)-\varphi[t](q)|]^{\prime}\left[2 \sin \left(\frac{\theta[t, j](p q)}{2}\right)\right][\mathbf{n}[t, j](p q) \cdot g[t, j](p q)] \\
& +\sum_{\langle p q\rangle \in \mathcal{T}_{1}(j)}[|\varphi[t](p)-\varphi[t](q)|]\left[2 \sin \left(\frac{\theta[t, j](p q)}{2}\right)\right]^{\prime}[\mathbf{n}[t, j](p q) \cdot g[t, j](p q)] \\
& +\sum_{\langle p q\rangle \in \mathcal{T}_{1}(j)}[|\varphi[t](p)-\varphi[t](q)|]\left[2 \sin \left(\frac{\theta[t, j](p q)}{2}\right)\right][\mathbf{n}[t, j](p q) \cdot g[t, j](p q)]^{\prime} .
\end{aligned}
$$


We assert that

$$
\frac{d}{d t}(\delta V[\mathcal{T}(j, t)](g[t]))=\sum_{\langle p q\rangle \in \mathcal{T}_{1}(j)} \pm C L^{3}=\sum_{\langle p q\rangle \in \mathcal{T}_{1}(j)} \pm C L(j)^{3} .
$$

To see this we will estimate each of the three summands above.

First summand We use 3.7, 3.10(2), 3.11(5) to estimate for each $p q$,

$$
\begin{gathered}
{[|\varphi[t](p)-\varphi[t](q)|]^{\prime}\left[2 \sin \left(\frac{\theta[t, j](p q)}{2}\right)\right][\mathbf{n}[t, j](p q) \cdot g[t, j](p q)]} \\
=\left(C L^{2}\right)(C L)\left(1 \pm C L^{2}\right) .
\end{gathered}
$$

Second summand We use 3.10(5), 3.11(7) to estimate for each $p q$,

$$
\begin{aligned}
& {[|\varphi[t](p)-\varphi[t](q)|]\left[2 \sin \left(\frac{\theta[t, j](p q)}{2}\right)\right]^{\prime}[\mathbf{n}[t, j](p q) \cdot g[t, j](p q)]} \\
& =[|\varphi[t](p)-\varphi[t](q)|][\theta[t, j](p q)]^{\prime} \\
& \quad+[|\varphi[t](p)-\varphi[t](q)|]\left[2 \sin \left(\frac{\theta[t, j](p q)}{2}\right)-\theta[t, j](p q)\right]^{\prime} \\
& \quad+[|\varphi[t](p)-\varphi[t](q)|]\left[2 \sin \left(\frac{\theta[t, j](p q)}{2}\right)\right]^{\prime}[\mathbf{n}[t, j](p q) \cdot g[t, j](p q)-1] \\
& =[|\varphi[t](p)-\varphi[t](q)|][\theta[t, j](p q)]^{\prime} \pm(C L)\left(C L^{2}\right) \pm(C L)(C)\left(C L^{2}\right) .
\end{aligned}
$$

Third summand We use 3.10(2) and 3.11(6) to estimate

$$
\begin{gathered}
{[|\varphi[t](p)-\varphi[t](q)|]\left[2 \sin \left(\frac{\theta[t, j](p q)}{2}\right)\right][\mathbf{n}[t, j](p q) \cdot g[t, j](p q)]^{\prime}} \\
=(C L)(C L)(C L) .
\end{gathered}
$$

According to Schlafli's formula [7],

$$
\sum_{\langle p q\rangle \in \mathcal{T}_{1}(j)}[|\varphi[t](p)-\varphi[t](q)|][\theta[t, j](p q)]^{\prime}=0 .
$$

Our assertion follows. 


\subsection{Main Theorem}

(1) For each fixed time $t$,

$$
\lim _{j \rightarrow \infty} \delta V[\mathcal{T}(j, t)](g[t])=\delta V_{t}(g[t]) .
$$

(2) For each fixed $j, \delta V\left[\mathcal{T}(j)_{t}\right](g[t])$ is a differentiable function of $t$ and

$$
\lim _{j \rightarrow \infty} \frac{d}{d t}\left(\delta V\left[\mathcal{T}(j)_{t}\right](g[t])\right)=0
$$

uniformly in $t$.

(3) For each $t$

$$
\int_{\mathcal{M}_{t}} H_{t} d \mathcal{H}^{2}=\int_{\mathcal{M}} H d \mathcal{H}^{2}
$$

This is the main result of this note.

Proof To prove the first assertion, we check that

$$
\left(\rho_{t}\right)_{\sharp} V[\mathcal{T}(j, t)]=V_{t}
$$

for each $t$ and all large $j$. Indeed, the $\tau$ regularity of our triangulations implies that the normal directions of the $\mathcal{N}\left[\mathcal{T}(j)_{t}\right]$ are very nearly equal to the normal directions of nearby points on $\mathcal{M}_{t}$ and that the restriction of $D \rho_{t}$ to the tangent planes of the $\mathcal{N}\left[\mathcal{T}(j)_{t}\right]$ is very nearly an orthogonal injection. The first assertion follows with use of the first variation formula given in [14.1, 4.2]. Assertion (2) follows from 4.1 since

$$
\sum_{\langle p q\rangle \in \mathcal{T}_{1}(j)} L(j)^{2}
$$

is dominated by the area of $\mathcal{M}$ (see 2.2.12) and $\lim _{j \rightarrow \infty} L(j)=0$. Assertion (3) follows from assertions (1) and (2) and our observation in 2.1.4.

Acknowledgements Fred Almgren tragically passed away shortly after this note was written. Since then, the main result for smooth surfaces has been reproved in an easier way and generalized to the setting of Einstein manifolds by J-M Schlenker together with the second author of the current paper [6]. Nonetheless, it seems clear that the methods used here can be used to extend these results in other directions. 


\section{References}

[1] W K Allard, On the first variation of a varifold, Annals of Math. 95 (1972) 417-491

[2] M Berger, B Gostiaux, Geometrie differentielle: varietes, courbes et surfaces, Presses Universitaires de France, III (1987)

[3] J Cheeger, W Müller, R Schrader, On the curvature of piecewise flat spaces, Comm. Math. Phys. 92 (1984) 405-454

[4] R Connelly, I Sabitov, A Walz, The bellows conjecture, Beiträge Algebra Geom. 38 (1997) 1-10

[5] G Herglotz, Ueber der Starrheit der Eiflachen, Abh. Math. Semin. Hansische Univ. 92 (1943) 127-129

[6] I Rivin, J-M Schlenker, Schläfli formula and Einstein manifolds, IHES preprint (1998)

[7] D V Alekseevsky, 亡̀ B Vinberg, A S Solodovnikov, Geometry of spaces of constant curvature, from: "Geometry II", Encyclopaedia Math. Sci. 29, SpringerVerlag, Berlin (1993)

[8] M Spivak, A Comprehensive Introduction to Differential Geometry, (Second Edition) Publish or Perish, Berkeley (1979)

Mathematics Institute, University of Warwick

Coventry, CV4 7AL, UK

Email: igor@maths.warwick.ac.uk

Received: 10 May 1998 